\title{
Virulence and pathogenicity aspects in Candida albicans infections
}

\section{Omar Sadik, Irina Gheorghe, Mariana Carmen Chifiriuc}

\author{
${ }^{1}$ Department of Microbiology, Faculty of Biology, University of Bucharest, Aleea Portocalelor No. 1-3, 060101, \\ Bucharest, Romania; ${ }^{2}$ Research Institute of the University of Bucharest (ICUB), Spl. Independentei 91-95, \\ 76201, Bucharest, Romania \\ ${ }^{\square}$ Correspondence to: Omar Sadik, Department of Microbiology, Faculty of Biology, University of Bucharest, Aleea \\ Portocalelor No. 1-3, 060101, Bucharest, Romania E-mail: omar_ss_82@yahoo.com
}

Received: 4 January 2018 / Revised: 26 March 2018 / Accepted: 30 March 2018 / Available online: 21 April 2018

\begin{abstract}
Candida albicans is a commensal yeast that often colonizes various parts of humans, mainly epithelial, dermal or mucosal (oral cavity, gastrointestinal and genital tract etc.) tissues, but also an opportunistic pathogen that can be involved in a wide range of asymptomatic to severe, invasive infections in immunodeficient individuals. It exhibits certain unique properties such as phenotypic switching (crucial for avoiding the immune system), unique mating pattern (both asexual and sexual) and haploinsufficiency. This fungus has been also known to be a major cause of oral and skin infections, with a higher number of cases occurring in HIV positive individuals. Strains isolated from different parts of the body exhibit certain differences in their morphology and properties, among which, biofilms formation ability needs to be better understood in order to design efficient antifungal strategies. This review paper gives a brief description of various aspects of Candida albicans pathogenesis.
\end{abstract}

Keywords: Candida albicans, opportunistic infections, phenotypic switching, fungal biofilms

\section{Introduction}

Candida albicans is present in the microbiota of $80 \%$ of the healthy individuals (Odds, 1988), but also, among the species of genus Candida, it is considered the most prevalent opportunistic fungal pathogen that could exhibit extreme virulence in humans (McCullough et al., 1996), particularly in immunocompromised individuals. The normal colonization turns to an infection usually when there is a change in the environment which shifts the conditions sustaining an excessive growth of the microorganism. C. albicans possesses several virulence factors that aid the yeast in escaping the host's immune system. The prominent virulence factors are phenotypic switching, adhesins and biofilm formation, as well as the production of soluble enzymes (Zanni et al., 2017). This paper aims to review the steps of $C$. albicans infections pathogenesis and the underlying virulence factors.

\section{Clinical aspects of $C$. albicans infections}

Candidiasis is a very common human infection which usually affects the oral cavity, esophagus, gastrointestinal tract, skin, vagina and even the vascular system. Four conditions can be termed as oral candidiasis: (1) pseudomembranous candidiasis (oral thrush) usually appearing as creamy white lesions on the oral mucosa. Most prominent diagnosing feature of this infection is that these plaques can be removed by gentle scraping. Examination of the scraped pseudomembranes reveals the presence of fungus (McCullough and Savage 2005; Reichart et al., 2000); (2) acute erythematous candidiasis. This form of infection usually occurs due to the lowering of the bacterial population in the mouth following the administration of broad spectrum antibiotics, giving the opportunity to $C$. albicans to grow and spread. The infection can occur on the mouth mucosa, but usually appears as reddened lesions on the dorsum of the tongue and also the palate (Reichart et al., 2000; Gableri et al., 2008). Also this infection is prominent in HIV positive and AIDS individuals (Hodgson and Rachanis, 2002); (3) chronic hyperplastic candidiasis (candida leukoplakia) can occur on any mucosal surface and it appears as a white, smooth or nodular lesions which cannot be removed by gentle scraping. It is usually prevalent in middle aged men who smoke and usually has tendency towards a malignant transformation (Holmstrup and Bessermann 1983; Arendorf et al., 1983; Cawson and Binnie 1980); 
(4) Candida-associated denture stomatitis, which is quite prevalent and does not reveal any symptom. It usually occurs in almost $65 \%$ of all the denture wearers, in which the stagnant area above the upper fitting surface of the denture serves as an ideal environment for the growth of Candida sp. cells (Webb et al., 1998; Radford et al., 1999).

In a study aiming to investigate the density of Candida sp. in periodontal pockets, healthy subgingival sites, and oral rinse samples in patients with untreated periodontitis, $C$. albicans was the prevalent species that was identified, being found in almost $46.7 \%$ of the periodontitis patients and 32\% of the healthy subjects. The Multilocus Sequencing Typing (MLST) method analysis revealed that certain specific periodontal sites are specifically colonized by distinct Candida species that are not found on the oral mucosa or in other periodontal pockets, suggesting some specific enrichment of particular isolates or species at these sites. (McManus et al., 2012).

Secondary forms of oral candidiasis also occur frequently and are known as Candida-associated lesions. Angular cheilitis is a common Candida-associated lesion where lesions occur at the angles of the mouth. Microbiological examination usually reveals the presence of $C$. albicans along with Staphylococcus aureus strains (Dias and Samaranayake, 1995). Another such secondary infection is the median rhomboid glossitis, which is a chronic condition. It usually appears as a diamond-shaped lesion at the posterior midline on the dorsum of the tongue (McCullough \& Savage, 2005). Some associations between $C$. albicans and linear gingival erythema have also been found. However, this condition is more commonly seen in HIV patients (Grbic et al., 1995; Velegraki et al., 1999). Other species of Candida genus have been known to cause some serious skin infections. Cutaneous candidiasis is caused by endogenous, saprophytic Candida blastopores which are selectively found in oral, gastrointestinal, vaginal, and cutaneous epithelium. Other studied species such as C. tropicalis, and $C$. parapsilosis did not revealed the ability of adherence to the human skin (Raz-Pasteur et al., 2011).

In HIV-infected patients the spectrum of $C$. albicans infections varies from asymptomatic to severe ones. In a study conducted on 165 HIV patients, it was observed that 80 patients had $C$. albicans infection, which was the most commonly observed (Anwar et al., 2012). Another study had reported that asymptomatic carriage of Candida species was quite common in case of HIV positive patients. A very high rate of oropharyngeal carriage of Candida was observed in HIV positive patients. Also it has been further revealed that persistent asymptomatic carriage of Candida species is often a possible risk factor for subsequent oral infections. In a study conducted in HIV-infected subjects in Nigeria, it was seen that almost $60 \%$ of the patients had oropharyngeal infection and $C$. albicans was the most predominant species (Nweze and Ogbonnaya, 2011).
Apart from C. albicans, another species that was identified in these patients was $C$. tropicalis. It has been demonstrated that the status of oral Candida carrier was not associated with the number of CD4+ lymphocytes or with the viral load (Costa et al., 2006). In another experiment, the researchers observed a low rate of oral Candida carriage in patients infected by HIV who were on highly active antiretroviral therapy (Ribeiro et al., 2015).

A number of 43 C. albicans isolates from animals, including mammals and avian species with diverse global distribution were compared with human isolates using MLST method. The analysis indicated that $C$. albicans isolates from animals showed a trend towards genetic separation from isolates that colonize and infect humans, while still maintaining some common traits, i.e. $C$. albicans isolates associated with animals undergo evolutionary change in the direction of greater adaptation to their non-human hosts (Jacobsen et al., 2008).

Studies have shown that pre-treatment with Lactobacillus sp. is quite successful in preventing C. albicans infection. They were successful in protecting human oral mucosa cells against damage caused by $C$. albicans infections and it was seen that inflammatory reactions were milder even without the treatment. It was found that after consuming yoghurt (containing L. acidophilus) there was a threefold decrease of vaginal candidiasis as compared to the control group (Cartwright, 2010).

In animal studies, the presence of probiotic bacteria (Lactobacillus acidophilus, L. reuteri, L. casei, or Bifidobacterium animalis) in the gastrointestinal tract prolonged the survival of adult and neonatal immunodeficient mice compared to that of isogenic mice which were colonized with $C$. albicans alone. It was also observed that the population of the fungus was significantly reduced in the case of mice treated with probiotic bacteria. Although the probiotics were not able to prevent candidiasis, however they were able to reduce the number and severity of the mucosal and systemic infections, to prolong survival of mice and modulate the immune responses (Wagner et al., 1997).

However, one noteworthy point was that the probiotic bacteria did not inhibit the growth of the fungus directly. Rather there was a competition with the fungus for the common nutrients and adhesion sites. The reduced ability to attach to and enter mucosal cells was one of the major factors largely responsible for the greatly reduced damage to the mucosal cells (Schaller 2015).

\section{Pathogenesis of C. albicans infections}

\section{Biofilm formation by C. albicans}

Biofilms usually serve as safe, protective niches for microorganisms, where they are safe from the action of host defence mechanisms, antimicrobials and of other treatment options. Extensive studies have revealed that biofilms have a heterogeneous architecture made of both 
cellular as well as non-cellular elements (Chandra et al., 2001). Biofilm formation takes place in three distinct stages: early, intermediate and maturation which however can be further subdivided into: initial adherence, proliferation, colonization, maturation and ultimately dispersion. First the fungal cells need to adhere to a suitable surface (cellular or inert such as medical devices). This initial attachment is mediated by both nonspecific mediators as well as specific fungal adhesins. This initial attachment is followed by cell division, proliferations and biofilm development. The biofilm formation begins with the majority of $C$. albicans cells present in the form of spores. These spores would slowly develop into microcolonies within a time period of 3-4 hours. This will be followed by appearance of thick tracks of fungal growth (due to cell growth and aggregation) within 11 hours. This will then be followed by appearance of non-cellular material or matrix, mainly composed of cell wall like polysaccharides (mannan and other glycans) which appears like a haze-like film that is covering the fungal microcolonies within 12-14 hours. This intermediate stage is followed by a maturation phase in which the amount of the extracellular material increases, strongly encasing the fungal bodies (yeast cells and hyphae). Thus, this extracellular material encasing the fungal cells constitutes the mature $C$. albicans biofilm (Chandra et al., 2001). The structural complexity of the biofilm seems to facilitate the influx of nutrients and the waste disposal (Ramage et al., 2005). Attachment of the pathogenic organism to the host cell proteins reduces chances of its clearance by the host immune system (McCullough et al., 1996).

\section{Phenotypic switching in C. albicans and underlying molecular aspects}

It is well known that phenotypic plasticity is a unique feature of many fungal species. $C$. albicans is an extremely versatile, dimorphic yeast that can survive as a commensal in various environments. In addition to usual switching from the yeast form to the various filamentous forms, C. albicans also undergoes a phenomenon termed as phenotypic switching. The phenotypic switching in $C$. albicans isolates was first observed 1985, and was defined as the capacity to undergo spontaneous, reversible transitions between a fixed numbers of colony morphologies (Soll, 1992). This refers to a visible change in the morphology of the colonies. A single cell can give rise to various types of colonies. It has been observed that this switching occurs spontaneously at frequencies well above those produced by point mutation and is reversible in nature. Further, a cell obtained from a distinct type of colony, when re-cultured, produces the same type of colony thus depicting that variant colony morphology, once formed, gives rise to the same type of colony. There are several attributes that enable $C$. albicans to rapidly change its morphology such as it can rapidly switch from a unicellular form or yeast to a multicellular form having filaments or hyphae. Low doses of UV radiation can stimulate phenotypic switching and this switching can also take place at the site of infection thus indicating that it has role in pathogenesis also. The two most common phenotypic switching observed are the white-opaque transition and 3153A-type switching. On researching the mechanisms that regulated the phenotypic switching it was found that defects in the SIR2 gene lead to high a frequency of phenotypic switching, while deletion of this gene leads to a high number of karyotypic changes. These results indicate that this gene is involved in phenotypic switching (Pérez-Martín et al., 1999). The hyphal growth phase is characterized by the expression of genes that code for proteins, adhesins and secreted aspartyl proteinases (SAP). Both genetic and chromatin modifying elements (transcription factors, DNA methylation, histone modifications, and chromatin remodelers etc.) are involved in the regulation of yeasthyphae transition. It can be thus concluded that $C$. albicans itself undergoes significant epigenetic changes while switching their phenotype from a commensal to a pathogen. White opaque transition refers to two distinct morphologies of the colonies. Apart from morphological distinctions, both of these colonies differ in their mating preferences, their ability to colonize, survive in specific host niches, and susceptibility to various host defense mechanisms (which include neutrophils and macrophages). All the evidence points in the direction that this switching evolved as a survival strategy to avoid assault from immune response. The acetylation level of H3K56 is critical in maintaining the opaque form. In addition, opaque cells have less chromatin bound histone $\mathrm{H} 3$ than the white cells across the promoter of several genes. Therefore, it is possible that, interplay between H3K56ac levels and H2A.Z deposition may be a key regulator in maintaining this morphological transition in C. albicans (Brown, 2002; Sasse et al., 2013; Stevenson and Liu, 2011; Rai et al., 2018).

Earlier it was stated that $C$. albicans exhibited only asexual mating and existed only in the form of an obligate diploid being thus considered that it is extremely sensitive to a $50 \%$ loss in gene product. However, it has been shown that haploinsufficiency is quite prevalent in C. albicans. This could be explained by a transfection effect that could be responsible for depressing gene expression from the intact copy of the gene when the other copy is deleted or has undergone a transposon insertion. Last but not the least, the two alleles of a gene could have differentiated encoding proteins that have subtly different functions (Uhl et al., 2003). Researchers have found that a total of 69 genes (representing 10\%), including 47 small and 22 large size mutants found in haploinsufficient $C$. albicans are important for virulence or adaptation in the human host. It was proposed that virulence defect in small size mutant could be linked to the reduced contact surface of $C$. albicans, with either host cells or medical devices in case of biofilm infections. In the genome wide survey it was found that out of a total of $300(43.8 \%)$ dispensable genes, only 87 
$(12.7 \%)$ essential genes were haploinsufficient for size. Overall, it was found that the conserved regulators (Sch9, Sfp1, Cln3) and biological processes (ribosome biogenesis and cell cycle control) were required to maintain size homeostasis in this opportunistic yeast.

There has been found a mating locus similar to that of sexually reproducing yeast and it was revealed that $C$. albicans strains could reproduce sexually too. Scientists also found that several aspects of $C$. albicans mating were unique for this pathogen. Further studies have indicated that $C$. albicans strains have developed mating in order to survive in certain specific regions of the host where mating must take place in less than optimum conditions (Bennett and Johnson 2005).

The phenotypic switching - white-opaque cells and the colony morphology change - were not frequently found among $C$. albicans isolated from HIV-infected patients and also from healthy individuals (Vargas et al., 2000). C. albicans strains isolated from HIV-positive patients indicated that they had a higher cell growth rate as compared to strains isolated from HIV-negative individuals.

In order to understand the phenotypic and genotypic relationships in $C$. albicans virulence, there has been conducted an experiment including two types of $C$. albicans strains, i.e. strains isolated from blood stream infections in hospitalized patients, referred as $\mathrm{S}$ (systemic) strain and strains isolated from women with vaginal infections diagnosed in a community clinic, designated as $\mathbf{M}$ (mucosal) strains. Adhesion property of these two types of strains were studied using two cell lines, human mouth epithelial cells (BEC) and human skin epithelial cell line-the HaCat cell line. As for the BEC cells, there was no difference in the adherence property of the two types of strains, however, M strains showed higher adherence capacity to HaCat cells. Production of hydrolytic enzymes was seen to be higher in the $S$ strains. The $S$ strains were prominently white type colony. All S strains were diploid and a half of the studied strains in this group had higher DNA content than the diploid control strain, with a statistical difference between the $\mathrm{S}$ and $\mathrm{M}$ groups (Mandelblat et al., 2017).

\section{Soluble enzymes involved in the pathogenesis of C. albicans}

There are a number of virulence factors such as exoenzymes - phospholipase, proteinase and DNA-se, and the exotoxin haemolysin that contribute to the fungus pathogenicity. It has been shown that $C$. albicans strains isolated from HIV patients had less hemolytic activity (Wibawa and Aman 2015). In a study performed on $C$. albicans isolates recovered from HIV-infected women undergoing HAART therapy and HIV - uninfected women, it was found that the strains from the HIVpositive women showed higher proteinase activity, lower capacity to form biofilms and germ tubes. Overall the obtained results indicate that the fungal strains isolated from HIV-positive women might have a lower virulence (de MeloRiceto et al., 2016). However, depending upon the different clinical samples, different levels of the soluble virulence factors have been observed. For example, in the samples obtained from the oral cavity of HIV-negative and -positive individuals the exoenzymes phospholipase, proteinase and DNA-se, and the exotoxin haemolysin were expressed at higher level in the HIVpositive patients, results which are contradictory to previous observations (de Paula Menezes et al., 2016).

\section{Conclusions}

C. albicans is a commensal organism and the most common fungal species found in/on living humans. Usually nonpathogenic, $C$. albicans can produce serious infections in immunosuppressed patients, being a prevalent cause of oral and skin infections. Adhesion to different viable and inert substrata and biofilms formation are important features of $C$. albicans infections. This yeast species also expresses phenotypic switching, a change in morphology and cell wall components which enables it to strongly adhere at substratum and escape detection and elimination by the immune system. Several environmental, genetic and epigenetic factors regulate the switching of the phenotype of this yeast. The intimate mechanisms of these pathogenic features need to be better understood in order to design efficient antifungal strategies.

\section{Acknowledgments}

We gratefully acknowledge the support of the staff in Department of Microbiology, Faculty of Biology and Research Institute of the University of Bucharest (ICUB) and the financial support of the Government of Iraq.

(C) The Author(s) 2018

Open Access This article is distributed under the terms of the Creative Commons Attribution 4.0 International License (http://creativecommons.org/licenses/by/4.0/) which permits unrestricted use, distribution, and reproduction in any medium, provided you give appropriate credit to the original author(s) and the source, provide a link to the Creative Commons license, and indicate if changes were made.

\section{References}

Anwar K.P., Malik A., Subhan K. H. 2012. Profile of candidiasis in HIV infected patients. Iran J. Microbiol. 4, 204-209.

Arendorf T.M., Walker D. M., Roll J. R., Newcombe R.G. 1983. Tobacco smoking and denture wearing in oral candidal leukoplakia. Br. Dent. J. 155, 340-343.

Bennett R.J., Johnson A.D. 2005. Mating in Candida albicans and the search for a sexual cycle. Annu. Rev. Microbiol. 59, 233-255.

Brown A.J.P. 2002. Expression of Growth Form-specific Factors During Morphogenesis in Candida albicans. 
ASM Press. 87-94.

Cartwright P. 2010. Can Probiotics Reduce Candida Infections? In: The Clinical Use of Probiotics. Barlow J. (Ed.), Probiotics International Ltd, United Kingdom, 21-24.

Cawson R.A., Binnie W.H. 1980. Candida, leukoplakia and carcinoma: a possible relationship. In: Oral premalignancy. Proceedings of the first Dow Symposium. Iowa. University of Iowa. 59-66.

Chandra J., Kuhn D.M., Mukherjee P.K., Hoyer L.L., McCormick T., Ghannoum M.A. 2001. Biofilm formation by the fungal pathogen Candida albicans: development, architecture, and drug resistance. $J$. Bacteriol. 183, 5385-5394.

Costa C.R., Cohen A.J., Fernandes O.F.L., Miranda K.C., Passos X.S., Souza L.K.H., Silva M.D.R.R. 2006. Asymptomatic oral carriage of Candida species in HIV-infected patients in the highly active antiretroviral therapy era. Rev. Inst. Med. Trop. S. Paulo. 48, $257-$ 261.

De MeloRiceto R.É.B., Borges A.S., de BritoRöder D.V.D., dos Santos Pedroso R. 2016. Evaluation of virulence factors of Candida albicans isolated from HIV-positive individuals using HAART. Arch. Oral Biol. 66, 61-65.

Dias A.P., Samaranayake L.P. 1995. Clinical, microbiological and ultrastructural features of angular cheilitis lesions in Southern Chinese. Oral Dis. 1, 43-48.

Gableri I.G., Barbosa A.C., Velela R.R., Lyon S., Rosa C.A. 2008. Incidence and anatomic localization of oral candidiasis in patients with AIDS hospitalized in a public hospital in Belo Horizonte, MG, Brazil. J. Appl. Oral Sci. 16, 247-250.

Grbic J.T., Mitchell-Lewis D.A., Fine J.B., Phelan J.A., Bucklan R.S., Zambon J.J., Lamster I.B. 1995. The relationship of candidiasis to linear gingival erythema in HIV-infected homosexual men and parenteral drug users. J. Periodontol. 66, 30-37.

Hodgson T.A., Rachanis C.C. 2002. Oral fungal and bacterial infections in HIV-infected individuals: an overview in Africa. Oral Dis. 8, 80-87.

Holmstrup P., Bessermann M. 1983. Clinical, therapeutic, and pathogenic aspects of chronic oral multifocal candidiasis. Oral Surg. Oral Med. Oral Pathol. 56, 388-395.

Jacobsen M.D., Bougnoux M.E., d'Enfert C., Odds F.C. 2008. Multilocus sequence typing of Candida albicans isolates from animals. Res. J. Med Microbiol. 159, 436440.

Mandelblat M., Frenkel M., Abbey D., Ben Ami R., Berman J., Segal E. 2017. Phenotypic and genotypic characteristics of Candida albicans isolates from bloodstream and mucosal infections. Mycoses 60, 534545.

McCullough M.J., Savage N.W. 2005. Oral candidosis and the therapeutic use of antifungal agents in dentistry. Aust. Dent. J. 50, S36-S39.
McCullough M.J., Ross B.C., Reade P.C. 1996. Candida albicans: a review of its history, taxonomy, epidemiology, virulence attributes, and methods of strain differentiation. Int. J. Oral Max. Surg. 25, 136144.

McManus B.A., Maguire R., Cashin P.J., Claffey N., Flint S., Abdulrahim M.H., Coleman D.C. 2012. Enrichment of multilocus sequence typing clade 1 with oral Candida albicans isolates in patients with untreated periodontitis. J. Clin. Microbiol. 50, 33353344.

Nweze E.I., Ogbonnaya U.L. 2011. Oral Candida isolates among HIV-infected subjects in Nigeria. J. Microbiol. Immunol. 44, 172-177.

Odds F.C. 1988. Candida and candidosis: a review and bibliography. 2nd ed, Bailliere Tindall, London, United Kingdom.

Pérez-Martín J., Uría J.A., Johnson A.D. 1999. Phenotypic switching in Candida albicans is controlled by a SIR2 gene. EMBO J. 18, 2580-2592.

Radford D., Challacombe S.J., Walter J.D. 1999. Denture plaque and adherence of Candida albicans to denturebase materials in vivo and in vitro. Critical Arch. Oral Biol. 10, 99-116.

Rai L.S., Singha R., Brahma P., Sanyal K. 2018. Epigenetic determinants of phenotypic plasticity in Candida albicans. Fungal Bıol. Rev. 32, 10-19.

Ramage G., Saville S.P., Thomas D.P., Lopez-Ribot J.L. 2005. Candida biofilms: an update. Eukaryot. Cell. 4, 633-638.

Raz-Pasteur A., Ullmann Y., Berdicevsky I. 2011. The pathogenesis of Candida infections in a human skin model: scanning electron microscope observations. ISRN Dermatol. 2011, Article ID 150642, 6 pages, doi:10.5402/2011/150642.

Reichart P.A., Samaranayake L.P., Philipsen H.P. 2000. Pathology and clinical correlates in oral candidiasis and its variants: a review. Oral Dis. 6, 85-91.

Ribeiro A.L.R., de AlencarMenezes T.O., de MeloAlvesJunior S., de Menezes S.A.F., Marques-da-Silva S.H., Vallinoto A.C.R. 2015. Oral carriage of Candida species in HIV-infected patients during highly active antiretroviral therapy (HAART) in Belém, Brazil. Int. J. Std. Alds. 120, 29-33.

Sasse C., Hasenberg M., Weyler M., Gunzer M., Morschhäuser J. 2013. White-opaque switching of Candida albicans allows immune evasion in an environment-dependent fashion. Eukaryot. Cell. 12, 5058.

Schaller M. 2015. How Lactobacillus bacteria fight Candida albicans infections. Retrieved from: https://www.gesundheitsindustriebw.de/en/article/news/how-lactobacillus-bacteria-fightcandida-albicans-infections/.

Soll D.R. 1992. High-frequency switching in Candida albicans. Clin. Microbiol. Rev. 5, 183-203.

Stevenson J.S., Liu H. 2011. Regulation of white and 
opaque cell-type formation in Candida albicans by Rtt109 and Hst3. Mol. Microbiol. 81, 1078-1091.

Uhl M.A., Biery M., Craig N., Johnson A.D. 2003. Haploinsufficiency-based large-scale forward genetic analysis of filamentous growth in the diploid human fungal pathogen C. albicans. EMBO J. 22, 2668-2678.

Velegraki A., Nicolatou O., Theodoridou M., Mostrou G., Legakis N.J. 1999. Paediatric AIDS-related linear gingival erythema: a form of erythematous candidiasis? J. Oral. Pathol. Med. 28, 178-182.

Wagner R.D., Pierson C., Warner T., Dohnalek M., Farmer J., Roberts L., Balish E. 1997. Biotherapeutic effects of probiotic bacteria on candidiasis in immunodeficient mice. Infect. Immun. 65, 4165-4172.

Webb B.C., Thomas C.J., Willcox M.D. P., Harty D.W.S., Knox K.W. 1998. Candida-associated denture stoma titis. Aetiology and management: A review. Part 2. Oral Dis. caused by Candida species. Aust. Dent. J. 43, 160-166.

Wibawa T., Aman, A.T. 2015. Virulence of Candida albicans isolated from HIV infected and non infected individuals. Springerplus 4, 408-418.

Zanni P.C.M.D., Bonfim-Mendonça P.D.S., Negri M., Nakamura S.S., Donatti L., Svidzinski T.I.E., Consolaro M.E.L. 2017. Virulence factors and genetic variability of vaginal Candida albicans isolates from HIV-infected women in the post-highly active antiretroviral era. Rev. Inst. Med. Trop. Sao Paulo. 5969. 